\title{
Cell-type specific regulation of cortical excitability through the allatostatin receptor system
}

\author{
Taruna Ikrar ${ }^{1+}$, Yulin Shi ${ }^{1 \dagger}$, Tomoko Velasquez ${ }^{3 \dagger}$, Martyn Goulding ${ }^{3 \dagger}$ and Xiangmin $\mathrm{Xu}^{1,2 * t}$ \\ Department of Anatomy and Neurobiology, School of Medicine, University of California, Irvine, CA, USA \\ 2 Department of Microbiology and Molecular Genetics, School of Medicine, University of California, Irvine, CA, USA \\ ${ }^{3}$ Molecular Neurobiology Laboratories, Salk Institute for Biological Studies, San Diego, CA, USA
}

\section{Edited by:}

Jessica Cardin, Yale University

School of Medicine, USA

Reviewed by:

Bing Zhang, University of

Oklahoma, USA

David J. Margolis, University of

Zurich, Switzerland

\section{*Correspondence:}

Xiangmin Xu, Department of

Anatomy and Neurobiology, School

of Medicine, University of California,

Irvine, CA 92697-1275, USA.

e-mail: xiangmin.xu@uci.edu

${ }^{\dagger}$ Author Contributions:

Experiments were conducted by

Taruna Ikrar and Yulin Shi;

Xiangmin Xu, Yulin Shi, and Taruna

Ikrar performed data analysis;

Tomoko Velasquez and Martyn

Goulding provided the AlstR mouse;

Xiangmin Xu wrote the paper.
Recent technical advances enable the regulation of neuronal circuit activity with high spatial and temporal resolution through genetic delivery of molecular activation or inactivation systems. Among them, the allatostatin receptor (AlstR)/ligand system has been developed for selective and quickly reversible silencing of mammalian neurons. However, targeted AlstR-mediated inactivation of specific neuronal types, particularly diverse types of inhibitory interneurons, remains to be established. In the present study, we achieved Cre-directed expression of AlstRs to excitatory and inhibitory cell-types in the cortex, and found that the AlstR-mediated inactivation was specific and robust at single-cell and neuronal population levels. Bath application of the allatostatin peptide markedly reduced spiking activity of AlstR-expressing excitatory and inhibitory neurons in response to intrasomatic current injections and laser photostimulation via glutamate uncaging, but control neurons without AlstR expression were not affected. As for the cortical network activity, the peptide application constrained photostimulation-evoked excitatory activity propagation detected by fast voltage-sensitive dye (VSD) imaging of the slices expressing AlstRs selectively in excitatory neurons, while it augmented excitatory activity in those slices with inhibitory neurons expressing AlstRs. In addition, AlstR-mediated inactivation effectively suppressed pharmacologically induced seizure activity in the slices targeting AlstRs to excitatory neurons. Taken together, our work demonstrated that the genetic delivery of AlstRs can be used for regulation of cortical excitability in a cell-type specific manner, and suggested that the AlstR system can be potentially used for fast seizure control.

Keywords: genetic inactivation, electrophysiology, photostimulation, voltage-sensitive dye imaging

\section{INTRODUCTION}

In the last decade, there has been considerable progress in the development and use of molecular and genetic methods for studying the central nervous system (Luo et al., 2008). Genetic strategies for activating or inactivating selected neurons have opened up new possibilities for understanding neuronal circuitry with high spatial and temporal resolution (Lechner et al., 2002; Boyden et al., 2005; Karpova et al., 2005; Tan et al., 2006; Armbruster et al., 2007; Lerchner et al., 2007; Chow et al., 2010). Along with other newly developed molecular techniques, the allatostatin receptor (AlstR) inactivation system can be a potent tool for regulating neocortical circuit activity with a high degree of specificity and effectiveness. The AlstR and its ligand peptide, allatostatin (AL), are found in insect brains, not native to mammalian neuronal tissue (Birgul et al., 1999). When expressed in mammalian neurons through genetic methods (Lechner et al., 2002; Gosgnach et al., 2006; Tan et al., 2006, 2008; Wehr et al., 2009), the receptor system can been used for silencing of neuronal activity as the application of the exogenous ligand quickly increases $\mathrm{K}^{+}$channel conductance and keeps membrane potentials from depolarizing above threshold through G protein-coupled AlstRs, which gate endogenous $G$ protein-coupled inward-rectifying $\mathrm{K}^{+}$(GIRK) channels in AlstRexpressing neurons. The AlstR/AL-mediated inactivation is estimated to occur within seconds to minutes, depending on the peptide delivery speed (Lechner et al., 2002; Tan et al., 2006). Endogenous expression of GIRK channels is common to the great majority of mature neurons in the mammalian brain (Karschin et al., 1996), such that cotransfection with GIRK channel subunits is unnecessary to effectively inactivate neurons using the AlstR/AL system (Lerchner et al., 2007; Wehr et al., 2009). Because mammals do not express the AL peptide or its receptor, peptide application should specifically inactivate only the neurons expressing the AlstR transgene.

Selective AlstR expression and its neuronal inactivation in the brains of mice, ferrets, and monkeys has been demonstrated via viral infection or using transgenic approaches (Gosgnach et al., 2006; Tan et al., 2006, 2008; Wehr et al., 2009). However, targeted AlstR-mediated inactivation of specific neuronal types, particularly diverse types of inhibitory interneurons in the neocortex, remains to be established. Given that there are multiple cell-types and each type is likely to have a unique role in cortical circuits, in the present study, we aimed to express the AlstR system to excitatory or inhibitory cell-types for cell-type 
selective regulation of cortical excitability through a double transgenic mouse approach. The transgenic mouse line conditionally expressing AlstR following Cre recombination (see below) was cross-bred with an Emx1-Cre mouse line (expressing Cre recombinase in most excitatory neurons of the cortex) (Guo et al., 2000; Kuhlman and Huang, 2008) or a Dlx5/6-Cre mouse line (expressing Cre in forebrain GABAergic neurons) for selective delivery of AlstRs to either excitatory or inhibitory cell-types in the brain.

We combined electrophysiology, laser scanning photostimulation, and fast voltage-sensitive dye (VSD) imaging to examine AlstR-mediated regulation of cortical excitability in living brain slice preparations, and addressed the following questions: (a) How effective is the Cre-mediated, selective expression of AlstRs? (b) Is the AlstR-mediated inactivation specific or robust at singlecell and neuronal population levels? (c) Can the AlstR system be used to suppress seizure activity when targeting AlstR expression to excitatory neurons? Our experiments demonstrated that the AlstR system can be effectively used for regulation of cortical excitability in a cell-type specific manner, and suggested that the AlstR system can be developed for potential translational applications in seizure control.

\section{MATERIALS AND METHODS SLICE PREPARATION}

All animals were handled and experiments were conducted in accordance with procedures approved by the Institutional Animal Care and Use Committee at the University of California, Irvine. In this study, we used a newer AlstR mouse line conditionally expressing AlstR and green fluorescent protein (GFP) following Cre recombination. This AlstR mouse line (R26-AlstR) was created by using the same targeting vector of transgene as the AlstR 192 strain (Gosgnach et al., 2006), but the vector was inserted at the Rosa26 locus to drive more stable expression. Specifically, the R26-AlstR mouse was first generated using a conditional double-stop cassette system that has been described previously (Kim et al., 2009). The AlstR gene followed by an IRES-EGFP cassette was placed downstream of the CAG promoter and FSFLSL double-stop cassette. The Cre-dependent R26-AlstR mouse line was then produced by removing the FSF stop cassette by crossing the original double-stop AlstR mouse with the mouse line of ACTB-FLPe transgene (Rodriguez et al., 2000). To achieve Cre-directed expression of AlstRs, the R26-AlstR mouse line was cross-bred with an Emx1-Cre mouse line (Guo et al., 2000) or a Dlx5/6-Cre mouse line (Monory et al., 2006). Emx1 is a mouse homologue of the Drosophila homeobox gene empty spiracles and its expression is largely restricted to excitatory neurons in the developing and adult cerebral cortex and hippocampus (Guo et al., 2000; Gorski et al., 2002; Kuhlman and Huang, 2008); the Emx1-Cre mouse line was created with the Cre gene placed directly downstream of the Emxl promoter (Guo et al., 2000). Dlx is a family of homeodomain transcription factors which are related to the Drosophila distal-less (Dll) gene (Panganiban and Rubenstein, 2002); in the Dlx5/6 Cre mouse, expression of Cre is directed to virtually all forebrain GABAergic neurons during embryonic development by the I56i and I56ii enhancers from the zebrafish dlx5a/dlx6a intergenic region (Monory et al., 2006).
To prepare living brain slices, the double transgenic mouse pups (postnatal day 16-21) or control pups (AlstR or Cre mouse pups) were deeply anesthetized with pentobarbital sodium (>100 mg/kg, i.p.) and rapidly decapitated, and their brains removed. For electrophysiological experiments, primary somatosensory (S1) cortical slices of $400 \mu \mathrm{m}$ thick were cut in sucrose-containing artificial cerebrospinal fluid (ACSF) (in mM: $85 \mathrm{NaCl}, 75$ sucrose, $2.5 \mathrm{KCl}, 25$ glucose, $1.25 \mathrm{NaH}_{2} \mathrm{PO}_{4}$, $4 \mathrm{MgCl}_{2}, 0.5 \mathrm{CaCl}_{2}$, and $\left.24 \mathrm{NaHCO}_{3}\right)$. Slices were first incubated in sucrose-containing ACSF for 30 minutes-one hour at $32^{\circ} \mathrm{C}$, and then transferred to recording ACSF (in mM: $126 \mathrm{NaCl}, 2.5 \mathrm{KCl}, 26 \mathrm{NaHCO}_{3}, 2 \mathrm{CaCl}_{2}, 2 \mathrm{MgCl}_{2}, 1.25$ $\mathrm{NaH}_{2} \mathrm{PO}_{4}$, and 10 glucose). For VSD imaging experiments, cortical slices were cut in ACSF with a broad-spectrum excitatory amino acid antagonist kynurenic acid $(0.2 \mathrm{mM})$. The ACSF with kynurenic acid was found to better preserve slice healthiness for dye staining than the sucrose-containing ACSF. After the initial incubation at $32^{\circ} \mathrm{C}$, slices were transferred for dye staining at room temperature for one hour in the ACSF and $0.1 \mathrm{mg} / \mathrm{ml}$ of the absorption VSD, NK3630 (Nippon KankohShikiso Kenkyusho Co. Ltd., Japan) and then maintained in the recording ACSF before use. Throughout the cutting, incubation, staining, and recording, the solutions were continuously supplied with $95 \% \mathrm{O}_{2}-5 \% \mathrm{CO}_{2}$.

\section{ELECTROPHYSIOLOGY, LASER SCANNING PHOTOSTIMULATION AND VOLTAGE-SENSITIVE DYE IMAGING}

Our overall system of electrophysiological recordings, photostimulation, and imaging was described previously (Xu et al., 2010; $\mathrm{Xu}, 2011)$. The solution was fed into the slice recording chamber through a pressure driven flow system with pressurized 95\% $\mathrm{O}_{2}-5 \% \mathrm{CO}_{2}$ with a perfusion flow rate of about $2 \mathrm{ml} / \mathrm{minute}$. To perform whole-cell recording, individual neurons were visualized at high magnification (60x objective), and were patched with glass electrodes of 4-6M $\Omega$ resistance that were filled with an internal solution containing (in $\mathrm{mM}$ ) $126 \mathrm{~K}$-gluconate, $4 \mathrm{KCl}$, 10 HEPES, 4 ATP-Mg, 0.3 GTP-Na, and 10 phosphocreatine ( $\mathrm{pH} 7.2,300-305 \mathrm{mOsm})$. The internal solution also contained $0.1 \%$ biocytin for cell labeling and morphological identification. Once stable whole-cell recordings were achieved with good access resistance (usually $<20 \mathrm{M} \Omega$ ), basic electrophysiological properties were examined through hyperpolarizing and depolarizing current injections. We focused on analyzing neuronal input resistance, spiking thresholds, and spike numbers at different steps of depolarizing current pulses in control ACSF, in the presence of AL, and after washout of AL. To examine AlstR-mediated inactivation, the ligand AL was added into the recording solution. Although nanomolar concentrations of AL could inactivated isolated cultured AlstR-expressing neurons (Lechner et al., 2002), $1 \mu \mathrm{M}$ AL (unless specified otherwise) were used for facilitating the ligand penetration deep into the slices and increasing temporal speeds of the ligand infusion. The AL application for 15 minutes was estimated to produce full AlstR effects, while the washout of 20 minutes was considered to remove the added AL from the recording solution.

During laser scanning photostimulation experiments, the microscope objective was switched from $60 \mathrm{x}$ to $4 \mathrm{x}$. Stock solution 
of MNI-caged-l-glutamate (Tocris Bioscience, Ellisville, MO) was added to $20 \mathrm{ml}$ of ACSF for a concentration of $0.2 \mathrm{mM}$ caged glutamate. The cortical slice image was acquired by a high resolution digital CCD camera, which in turn was used for guiding and registering photostimulation sites. A laser unit (DPSS Lasers, Santa Clara, CA) was used to generate $355 \mathrm{~nm}$ UV laser for glutamate uncaging. Short pulses of laser flashes (1 ms, $20 \mathrm{~mW}$ ) were controlled by using an electro-optical modulator and a mechanical shutter. The laser beam formed uncaging spots, each approximating a Gaussian profile with a width of $\sim 100 \mu \mathrm{m}$ laterally at the focal plane. During the experiments examining neuronal excitation profiles, photostimulation was applied to $8 \times 8$ patterned sites (with an inter-site space of $50 \mu \mathrm{m}^{2}$ ) centered at the recorded neurons in a non-raster, non-random sequence to avoid revisiting the vicinity of recently stimulated sites, while current-clamp recordings measured membrane potential depolarization and spiking activity. The neuronal excitation profile was simply assessed by the number of spiking locations to the photostimulation of $8 \times 8$ sites. Laser scanning photostimulation permits rapid evaluation of multiple stimulation locations with no physical damage to the tissue under our experimental conditions.

During VSD imaging experiments, a $705 \mathrm{~nm}$ light transilluminated brain slices and voltage-dependent changes in the light absorbance of the dye were captured by the MiCAM02 fast imaging system (SciMedia USA Ltd, Costa Mesa, CA). The illumination was supplied by optically filtering white light produced by an Olympus tungsten-halogen lamp up to $100 \mathrm{~W}$ of power. Optical recording of VSD signals was performed under the $2 \mathrm{x}$ objective with a sampling rate of 2.2 or $4.4 \mathrm{~ms}$ per frame (frame resolution $88(\mathrm{w}) \times 60(\mathrm{~h})$ pixels). The field of view covered the area of $2.56 \times 2.14 \mathrm{~mm}^{2}$ with a spatial resolution of $29.2 \times 35.8 \mu \mathrm{m}^{2} /$ pixel. For the imaging of photostimulationevoked excitatory activity with and without the AL application, VSD imaging of evoked activity was triggered and synchronized with each laser photostimulation at specified cortical sites. For each trial, the VSD imaging duration was 2000 frames including 500 baseline frames with an inter-trial interval of 12-14 seconds. To image pharmacologically induced spontaneous seizure-like activity, ACSF with $10 \mu \mathrm{M}$ bicuculline were infused into the slices for 15 minutes before VSD imaging; the imaging experiments consisted of seven sessions each at three perfusion conditions: bicuculline only, bicuculline $+\mathrm{AL}$, and bicuculline only again in ACSF. Each session had nine consecutive trials conducted with three minutes of off-illumination intervals between sessions. The number of seizure occurrences per session was measured under different conditions.

VSD signals were originally measured by the percent change in pixel light intensity $[\Delta I / I \%$; the $\%$ change in the intensity $(\Delta I)$ at each pixel relative to the initial intensity $(I)]$. In addition, the mean and standard deviation (SD) of the baseline activity of each pixel across the 50 frames before the spontaneous activity onset or preceding photostimulation was calculated, and VSD signal amplitudes were then expressed as SD multiples above the mean baseline signal for display and quantification. The activated pixel was empirically defined as the pixel with the amplitude $\geq 1$ SD above the baseline mean of the corresponding pixel's amplitude (equivalent to the detectable signal level in the original VSD maps of $\Delta I / I \%)$. VSD images were smoothed by convolution with a Gaussian spatial filter (kernel size: five pixels; SD $(\sigma)$ : one pixel), and a Gaussian temporal filter (kernel size: three frames; $\delta$ : one frame). In the present study, single-trial VSD signals were of sufficiently high amplitudes and could be discerned from background noise; no averaging over multiple trials was used for data presentation unless specified. To examine AlstR-mediated effects on photostimulation-evoked VSD responses, the total numbers of activated pixels and response amplitudes across the 10 frames around the peak VSD activation (e.g., at $55 \mathrm{~ms}$ after photostimulation) were measured for comparison across control, AL application, and washout. Images were displayed and analyzed using custom-made MATLAB programs.

\section{STATISTICAL ANALYSIS}

For statistical comparisons across more than two groups, we used the Kruskal-Wallis test (non-parametric One-Way ANOVA) and the Mann-Whitney $U$-test for group comparisons. Alpha levels of $p \leq 0.05$ were considered significant. All the values were presented as mean $\pm \mathrm{SE}$.

\section{RESULTS \\ SELECTIVE EXPRESSION OF AIstRs AND CHARACTERIZATION OF AlstR-MEDIATED NEURONAL INACTIVATION}

In the present study, we used Cre-directed expression of the AlstR transgene to excitatory and inhibitory cortical neurons by crossing the R26-AlstR mouse line conditionally expressing AlstR and GFP following Cre recombination (Gosgnach et al., 2006) with the Emx1-Cre mouse line (Guo et al., 2000) or the Dlx5/6-Cre mouse line (Monory et al., 2006). We confirmed the specificity and efficiency of these Cre mouse lines in achieving cell-type specific gene expression. As demonstrated in Figures 1A-F, through the use of a tdTomato Cre reporter line (Madisen et al., 2010), the Emx1-Cre mouse expresses Cre in a majority of excitatory pyramidal neurons of the cortex (Guo et al., 2000; Gorski et al., 2002) (Figures 1A-C), while the Dlx5/6 mouse expresses Cre specifically in inhibitory interneurons (Monory et al., 2006; Chao et al., 2010) (Figures 1D-F).

Emx1-cre: AlstR mice expressed AlstRs with GFP in cortical excitatory neurons, which was verified through GABA immunostaining (Figures 1G,H) and electrophysiological recordings (see below). Immunochemical examinations and living slice observations indicated that about $50 \%$ of excitatory cortical neurons express AlstRs, detected by their strong GFP appearance. Similarly, AlstR expression in inhibitory cortical neurons of Dlx5/6-Cre: AlstR mice was strong, as the native fluorescence enabled direct visualization and targeted electrophysiological recordings of the labeled neurons.

We started to test whether AlstR expression through the transgenic mouse approach led to functional inactivation of neuronal activity with single-cell electrophysiology. As illustrated in Figures 2A-D, consistent with GIRK activation, bath application of AL significantly decreased neuronal excitability of AlstR-expressing excitatory cells in response to somatic 


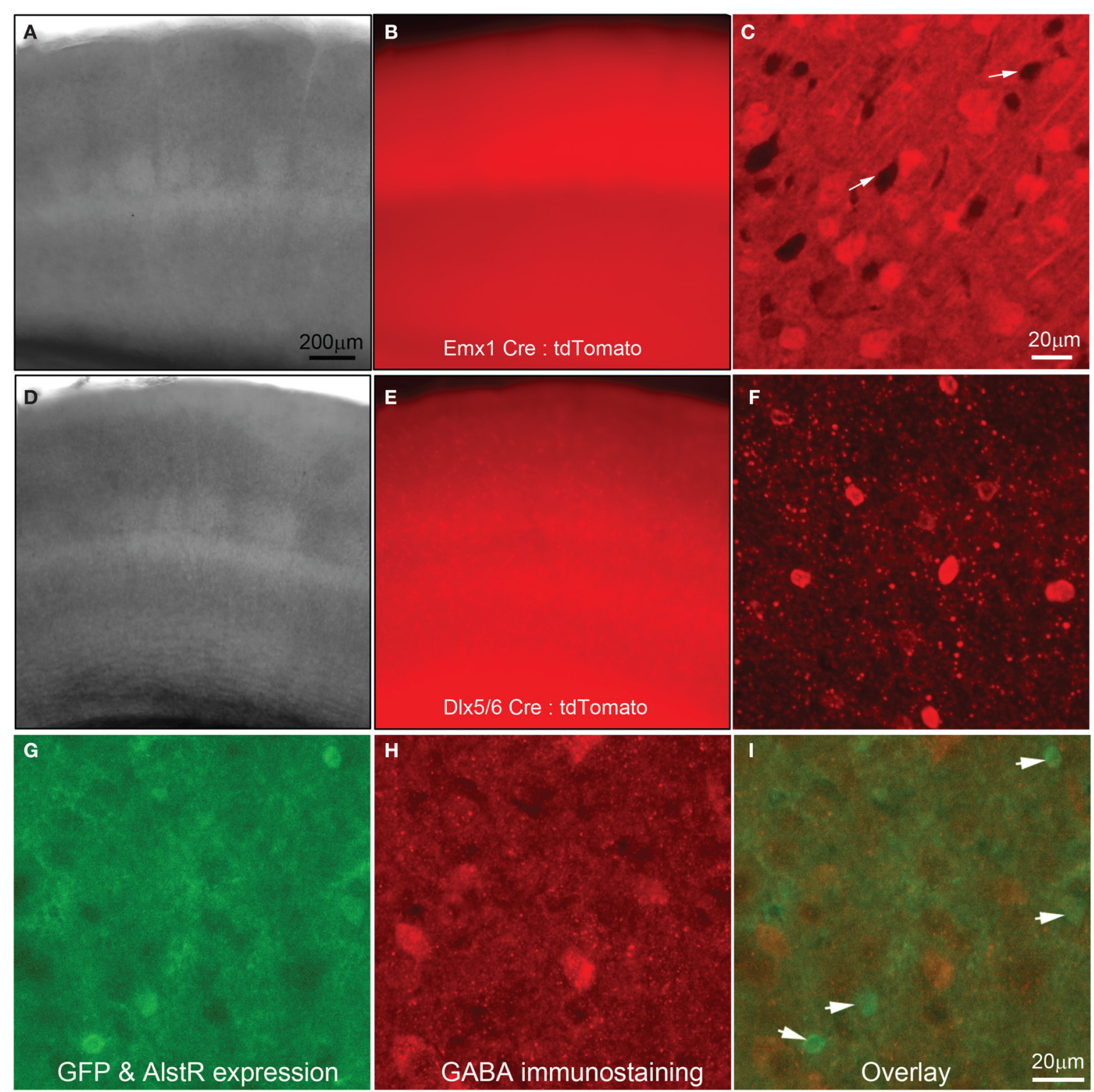

FIGURE 1 | Use of Cre-driver lines for targeted expression of allatostatin receptors (AlstRs) to excitatory or inhibitory cell-types. (A-C) Data

images from primary somatosensory (S1) cortex of a double transgenic mouse obtained by crossing the Emx1-Cre mouse line (Guo et al., 2000) to a Rosa-CAG-LSL-tdTomato Cre reporter line (Madisen et al., 2010). (A) and (B) are lower power images of a living cortical slice in bright field and epi-fluorescent mode. (C) Confocal microscopy of tdTomato-expressing neurons (presumptive excitatory neurons) under higher magnification. The white arrows point to the dark areas which may represent the somata of inhibitory cortical neurons. (D-F) Data images from S1 cortex of a double transgenic mouse by crossing the Dlx5/6-cre mouse line (Monory et al., 2006) to the tdTomato Cre reporter line. The camera exposure times for Emx1-Cre: tdTomato and DIx5/6-Cre: tdTomato fluorescent images were at an order of difference due to an extremely high-level of fluorescence in Emx1-Cre: tdTomato slices. (F) Confocal microscopy of tdTomato-expressing neurons (presumptive inhibitory neurons) under higher magnification. Note the morphological differences of tdTomato-expressing cells in (C) and (F). (G-I) AlstR-expressing neurons, resulting from crossing of the Emx1-Cre mouse line to the AlstR mouse line, are not immunopositive for GABA. In (G), the AlstR-expressing neurons also express GFP. $(\mathbf{H})$ is the same field of view of the slice with GABA immunostaining. (I) The overlay of the $(\mathbf{G})$ and $(\mathbf{H})$, with the arrowheads pointing to the GFP-AlstR-expressing neurons that are not GABA immunopositive. current injections in Emx1-Cre: AlstR mouse slices. Similar to previous findings (Lechner et al., 2002; Wehr et al., 2009), bath application of AL hyperpolarized resting membrane potentials, markedly reduced membrane input resistance of
AlstR-expressing (AlstR+) neurons (Figures 2B,D), and inhibited action potential firing by the decreased spike numbers elicited across a range of depolarizing current injections (Figures 2B,C). Control neurons without AlstR expression were 

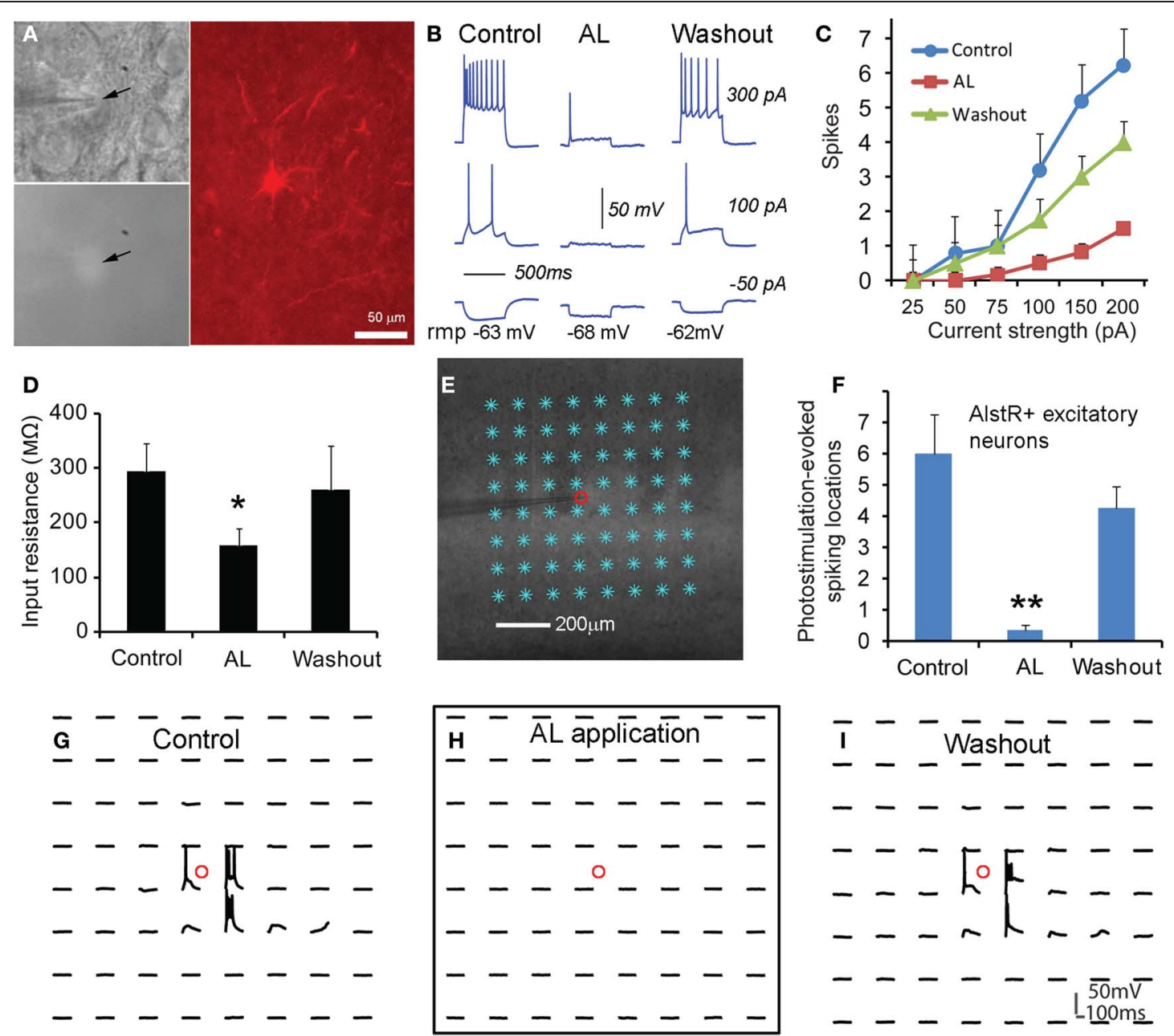

FIGURE 2 | Excitatory neuronal inactivation through the AlstR system. (A) One recorded excitatory neuron expressing GFP and allatostatin receptors in a Emx1-Cre: AlstR cortical slice. AlstR-expressing neurons were targeted based upon their GFP fluorescence (see the arrowheads), and identified post hoc as pyramidal cells based upon their morphology revealed by biocytin staining. (B) The AL application hyperpolarized the cell's resting membrane potential, reduced the cell's membrane input resistance, and suppressed action potential firing by intrasomatic current injections. rmp: resting membrane potential. The average hyperpolarization of excitatory neuronal resting membrane potentials was $-2.45 \pm 0.78 \mathrm{mV}$ ( $N=6$ cells). (C,D) Group data of significantly decreased intrinsic membrane excitability and input resistance in AlstR-expressing (AlstR+) excitatory neurons ( $N=6$ cells) in the presence of $A L$, respectively. (E) Illustration of the use of laser scanning photostimulation to assess evoked neuronal excitability with the slice image superimposed with photostimulation sites $\left(8 \times 8\right.$ sites, cyan $*, 50 \mu \mathrm{m}^{2}$ apart). The small red circle indicates the recorded cell location. (F) Group data ( $N=5$ cells) of AlstR-mediated suppression of photostimulation-evoked excitability measured by the number of spiking locations. (G-I) Photostimulation-evoked activity of the recorded neuron in (E) during control, AL application, and washout, respectively. The AL application totally abolished photostimulation-evoked spiking activity. In (D) and (F), * ${ }^{* *}$ indicate statistical significance between control and AL application of $p<0.05$ and $<0.005$, respectively. not affected $(N=10)$. Then we further characterized AlstRmediated inhibition of evoked excitability by laser scanning photostimulation. Compared to the examination of intrinsic membrane excitability through current injections, the measurement of photostimulation-evoked neuronal excitability via glutamate uncaging is more physiologically meaningful as the evoked excitability depends on the structure and membrane characteristics of the recorded neurons, and reflects the combination of intrinsic neuronal excitability and local synaptic input. Caged glutamate was present in the bath solution, and only turned active through focal UV photolysis. As shown in Figure 2E, we recorded from individual neurons and photostimulation was applied across multiple sites. Our data established that the AlstR-mediated inactivation was sufficiently strong and it essentially abolished the photostimulation-evoked spiking activity of the recorded AlstR-expressing excitatory neurons (Figures 2F-I).

As for inhibitory cortical neurons, we showed that the AlstR system produced robust inhibition on major subtypes of inhibitory cortical neurons through examinations of AlstRexpressing inhibitory neurons in Dlx5/6-Cre: AlstR mouse slices. The electrophysiological phenotype of the inhibitory neurons 
examined was fast-spiking, adapting-spiking, low-threshold spiking, or late-spiking; the cells had morphologies resembling basket cells, Martinotti cells, bitufted/bipolar cells, or neurogliaform cells. Compared to excitatory neurons, bath application of AL had a much larger hyperpolarizing effect on the resting membrane potentials of AlstR-expressing inhibitory neurons (Figures 3A,B). $\mathrm{AL}$ application consistently inhibited all the inhibitory neurons tested by decreasing their intrinsic excitability to current injections (Figure 3C) and photostimulation-evoked excitability (Figures 3D-L). It should be noted that the level of AlstRmediated inhibition varied with cell-types. For example, the spiking activity of AlstR+, fast-spiking cells tended to be suppressed completely while AlstR+, low-threshold spiking cells had remaining spikes in the presence of AL (Figures 3E-L). However, the overall effect of inhibition was substantial across interneuronal subtypes (Figures 3C,D).

\section{AlstR-MEDIATED EFFECTS ON NEURONAL POPULATION ACTIVITY}

After the examination of AlstR-mediated effects at the level of individual neurons, we went further to characterize the AlstR-mediated inhibition on neuronal population activity with our newly developed functional imaging technique. This is important because cortical circuits ultimately operate at the neuronal population level. Optical imaging with fast VSD was used to examine photostimulation-evoked population activity and to evaluate AlstR-mediated inhibition on cortical network activity. The VSD imaging can monitor neuronal activity in a large area simultaneously (Xu et al., 2010; Xu, 2011), with changes in optical absorption closely correlating with neuronal membrane depolarization. The brain slices were stained with VSD and perfused with ACSF containing caged glutamate. Then local cortical regions were stimulated with laser photostimulation, and circuit activation and output directly visualized by fast VSD imaging. For
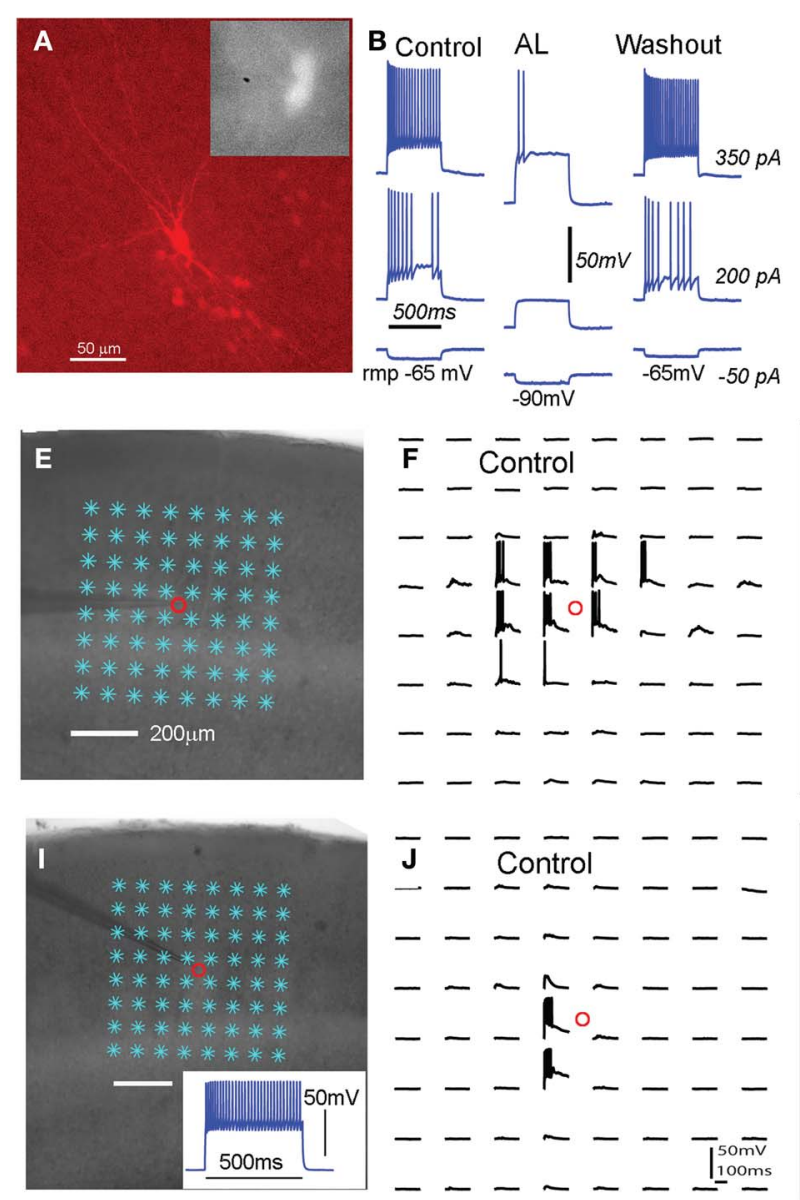

FIGURE 3 | Robust AlstR-mediated inhibition of diverse subtypes of inhibitory neurons. (A) shows one recorded inhibitory neuron expressing GFP and allatostatin receptors in the DIx5/6-Cre: AlstR cortical slice. This cell exhibited a low-threshold-spiking phenotype with bitufted morphology. (B) and (C) shows the $A L$ application caused a significant decrease in intrinsic neuronal excitability of the examined AlstR-expressing (AlstR+) inhibitory neurons $(N=12)$. The average hyperpolarization of inhibitory neuronal resting membrane potentials was $-11.4 \pm 0.94 \mathrm{mV}$. (D) shows the group data
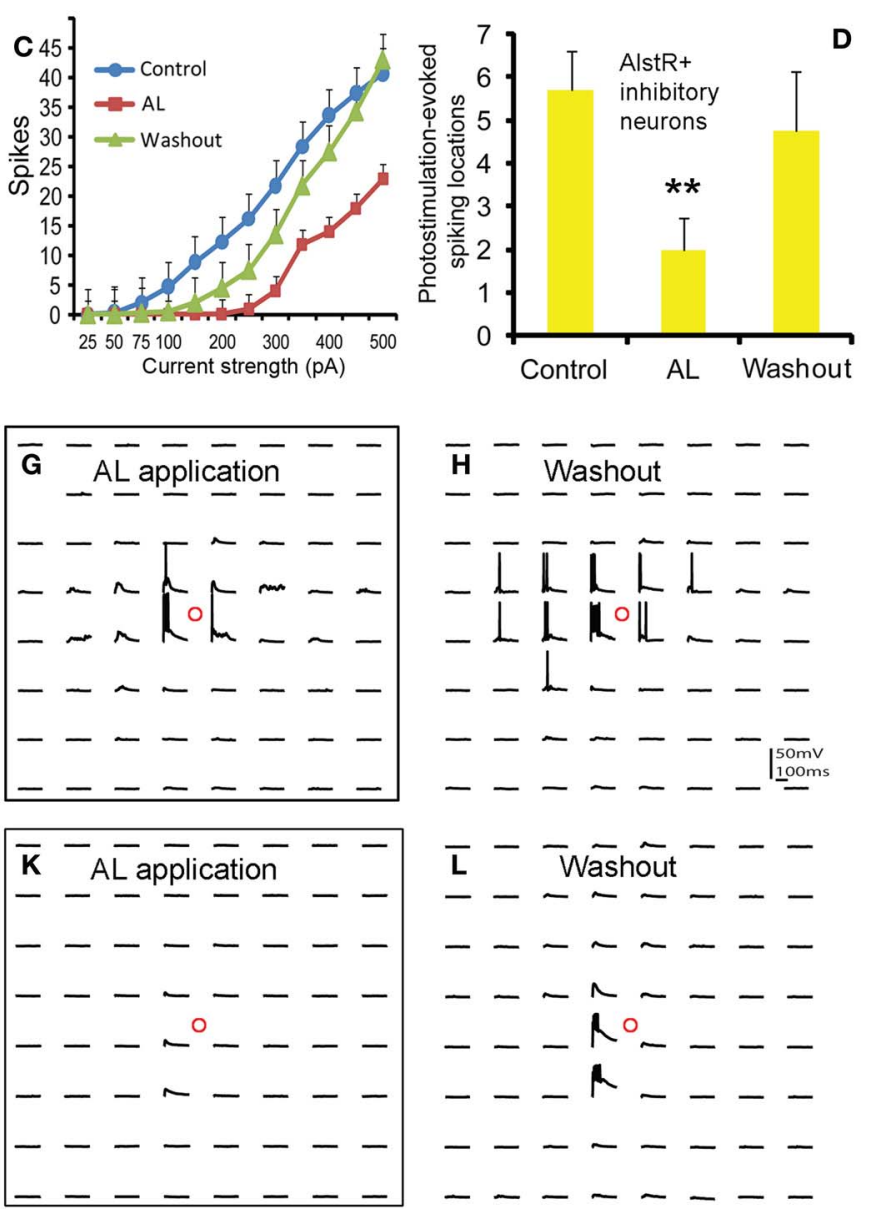

$(N=9)$ of AlstR-mediated suppression of photostimulation-evoked excitability measured by the number of spiking locations. (E-H) and (I-L) shows photostimulation-evoked activity of the recorded low-threshold-spiking neuron and a fast-spiking inhibitory neuron during control, AL application, and washout, respectively. The AL application substantially suppressed photostimulation-evoked spiking activity of AlstR-expressing inhibitory neurons. In (D), **indicates statistical significance of $p<0.005$ between control and $\mathrm{AL}$ application. 
the purpose of visual display, in Figure 4 and subsequent figures, the color scale codes the strength of excitatory activity (reflecting membrane potential depolarization of neuronal ensembles) with warmer colors indicating greater excitation. In addition, the time course of VSD signal (in the percent change of pixel intensity $[\Delta I / I \%])$ from the specified region of interest (ROI) is shown with the image data.

AlstR-mediated inhibition of excitatory neuronal population activity was found to be effective across different cortical sites (Figure 4). In Emx1-Cre: AlstR mouse slices, under control conditions, photostimulation in local cortical regions initiated excitation around $5 \mathrm{~ms}$ after laser exposure, which quickly propagated to functionally connected cortical regions within $50 \mathrm{~ms}$ in a columnar manner. Stimulation in the middle cortical layer (layer 4 or layer $5 \mathrm{a}$ ) caused excitatory activity to spread vertically to layers $2 / 3,5$, and 6 (Figures 4A1,A2 and 5A1,A2). Although photostimulation-evoked excitatory activity occurred in the presence of the AL peptide, evoked responses of the AlstR-expressing brain slice was clearly reduced and constrained (Figures 4B1,B2). Given that about half of excitatory cortical neurons expressed AlstRs in these slices, the AlstRmediated inhibition of excitatory population activity was quite effective. The AL effect was reversible with the peptide washout (Figures 4C1,C2). In comparison, AL application did not have effects on photostimulation-evoked population activity in the cortical slices without AlstR expression $(N=4)$. The reduction of VSD activation with the AL application for Emx1-Cre: AlstR slices, compared to control, is summarized in Figure 6A. On average, the total activation size during $\mathrm{AL}$ application was $18 \% \pm 2.0 \%$ of the control condition; the total response amplitude during $\mathrm{AL}$ application was $15.8 \% \pm 1.8 \%$ relative to control.

Conversely, we found that AlstR-mediated inactivation of GABAergic neurons augmented excitatory population activity. Photostimulation-evoked response in the presence of $\mathrm{AL}$ was dramatically increased in Dlx5/6-Cre: AlstR mouse slices (Figures 5A1,A2 and B1,B2), and the effect was reversed with the AL washout (Figures $\mathbf{5 C 1}, \mathbf{C 2}$ ). In agreement with the previous finding that $\mathrm{S} 1$ barrels are functionally independent cortical columns (Laaris et al., 2000; Petersen and Sakmann, 2001), in
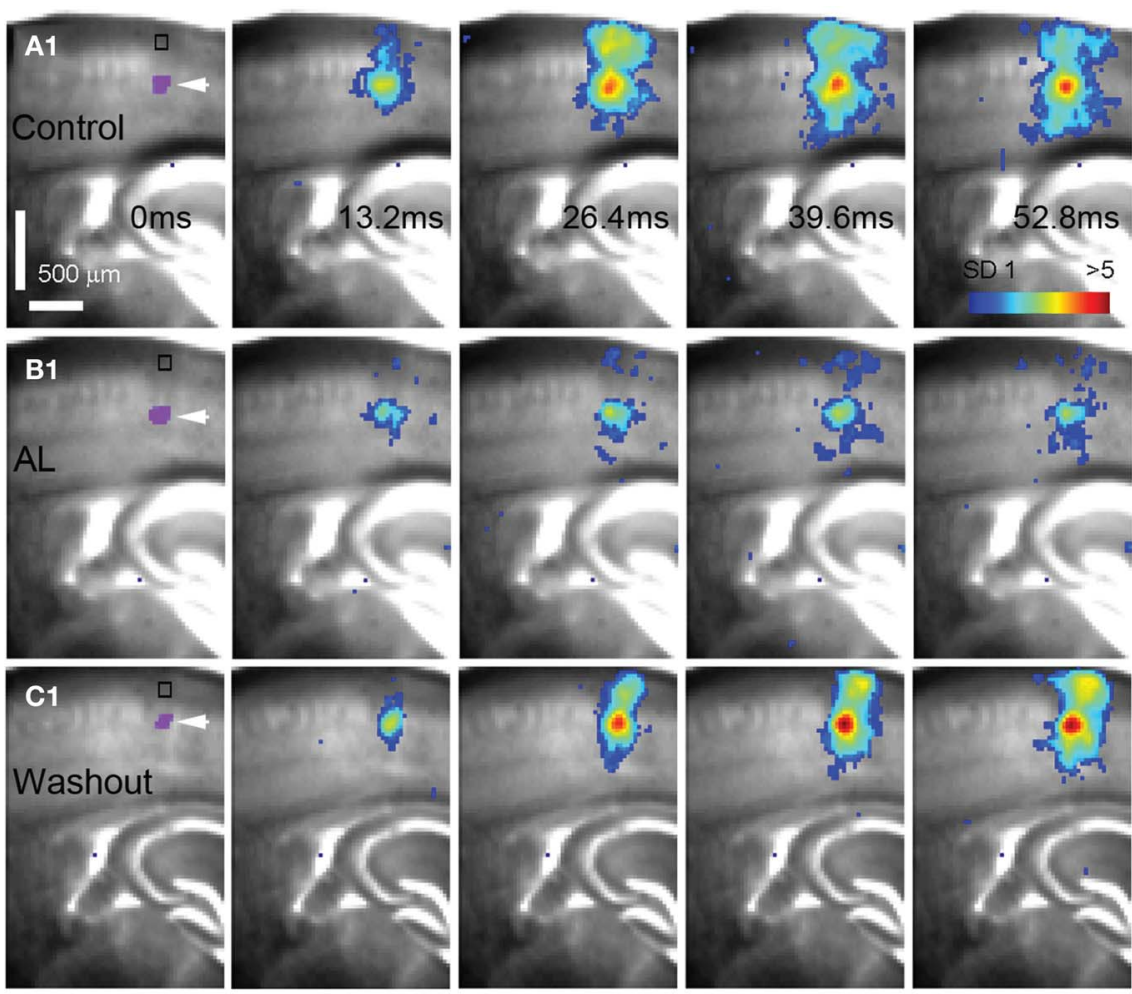

A2
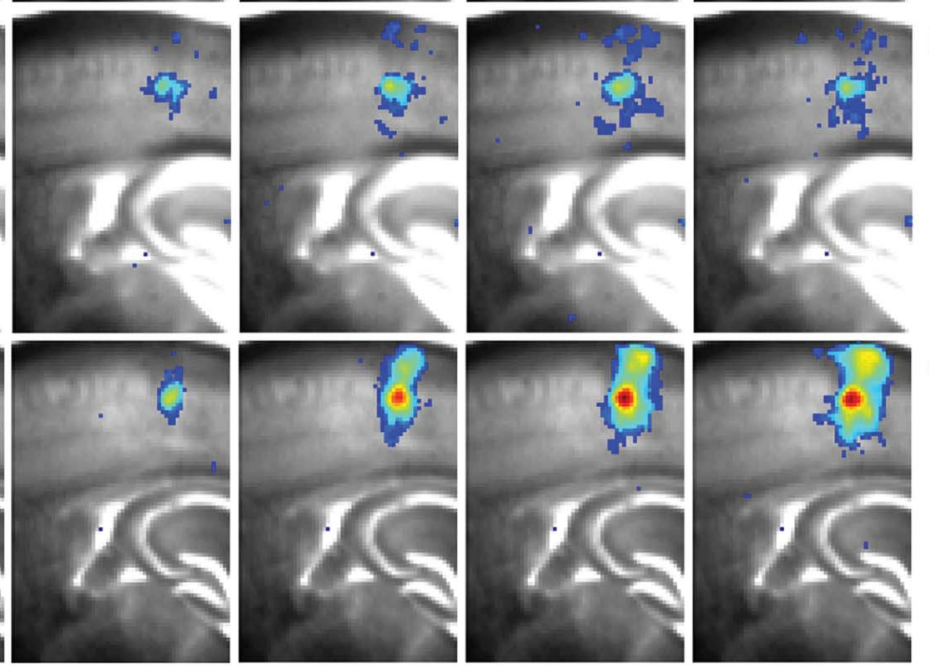

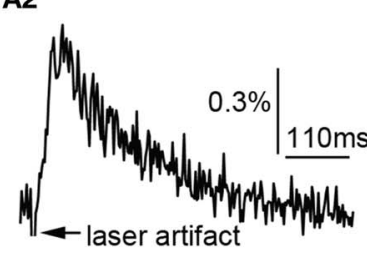

B2

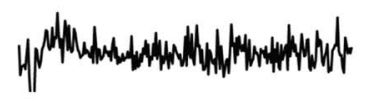

C2

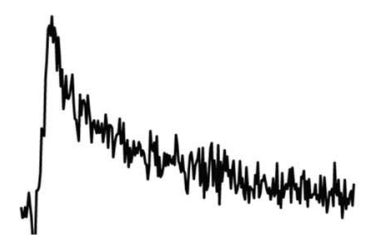

FIGURE 4 | AlstR-mediated inhibition of excitatory neuronal population activity in an Emx1-Cre: AlstR mouse slice. (A1) Time series data of VSD imaging of photostimulation-evoked activity in S1 cortex in normal ACSF. The color scale codes VSD signal amplitude expressed as standard deviation (SD) multiples above the mean baseline. The site of photostimulation between layers 4 and $5 a$ can be identified by the laser excitation artifact (purple) in the first frame, which is also indicated by the white arrowhead. Color-coded excitatory activity is superimposed on the background slice image. (A2) The time course of VSD signal (in the percent change of pixel intensity $[\Delta / / / \%]$ ) from the region of interest (ROI) indicated by the small rectangle in the first image frame in (A1). The arrowhead point the downward signal deflection caused by the laser excitation artifact. (B1) Time series of network activity in the same slice in the presence of the AL peptide, with excitatory response being clearly reduced compared to control. (B2) The time course of VSD signal (in the percent change of pixel intensity $[\Delta / / / \%]$ ) from the ROI indicated by the small rectangle in the first image frame in (B1). (C1) Time series of network activity in the same slice after the washout of the $A L$ peptide. (C2) The time course of VSD signal (in the percent change of pixel intensity $[\Delta / / / \%]$ ) from the $\mathrm{ROI}$ indicated by the small rectangle in the first image frame in (C1). 
normal ACSF, the lateral extent of photostimulation response in S1 layer 4 appeared to be limited to the cortical column defined structurally by the barrel (Figure 5A1). Yet, the importance of inhibitory neuronal regulation of cortical excitatory information flow is evident, as inhibition of GABAergic interneurons via the AlstR system caused the spread of excitation laterally in layer 4 as well as in wide regions of upper and deeper layers (Figure 5B1). Across the slices, the increase of VSD activation in the AL presence, compared to control, was $500.5 \% \pm 171 \%$ and $621.2 \% \pm 272.6 \%$ for the total activation size and response amplitude, respectively (Figure 6B).

\section{THE USE OF AIstR SYSTEM FOR SUPPRESSING SEIZURE ACTIVITY}

Given that targeted expression of AlstRs in excitatory neurons can strongly reduce cortical excitability with the ligand present, we were interested in investigating whether the AlstR system could be used for controlling seizure activity that is characterized by abnormal, excessive, or synchronous excitatory neuronal activity in the brain. We performed the experiments in single Emx1cre: AlstR slices sequentially. First, bath application of bicuculline induced spontaneous seizure-like activity in the slices. The epileptiform activity detected by VSD imaging, occurred in a small region and evolved into large propagating activity across the whole slice lasting for about 200-300 ms per event (Figures 7A1-A4 and B1,B2). After stable occurrences of the seizure activity across seven imaging sessions (126 seconds per session), AL peptide was co-applied with bicuculline and seizure occurrences continued to be monitored by VSD imaging. As illustrated in Figures 7C,D, the tendency of reduced seizure occurrences was clearly visible during the $\mathrm{AL}$ application. Note that the average durations or peak amplitudes of individual events during control and the AL infusion did not differ. Finally, the removal of AL from bath perfusion increased seizure occurrences, which could get back to the normal control level. For the Emx1Cre: AlstR mouse slices tested, the average number of seizure occurrences per minute was $1.09 \pm 0.14,0.41 \pm 0.12,0.68 \pm 0.38$ for bicuculline-containing ACSF, co-application of bicuculline and AL, and bicuculline-containing ACSF again, respectively. The co-application of AL with bicuculline reduced the occurrence of bicuculline-induced seizure activity significantly (Figure 7E). Thus, these data suggest that the AlstR system can be used to effectively suppress seizure activity.

\section{DISCUSSION}

In this study, we used a Cre-dependent transgenic mouse approach to effectively express AlstRs to specific types of cortical
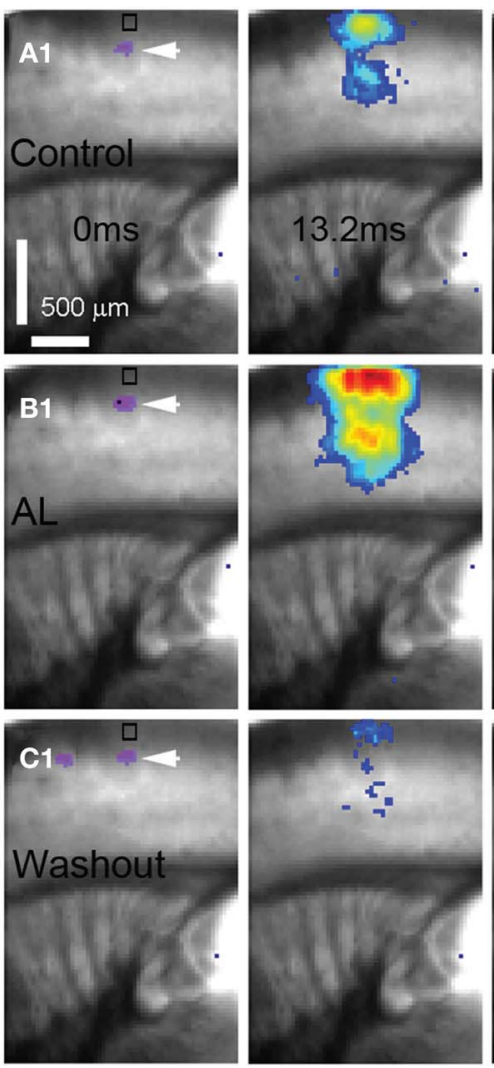
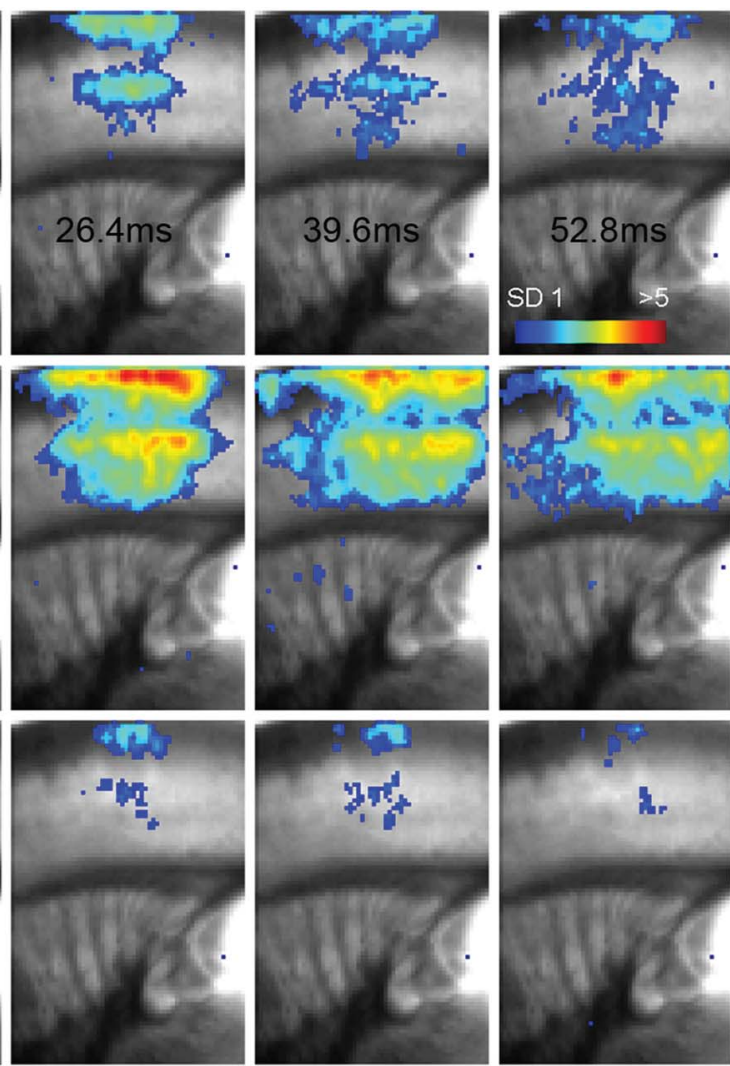

A2
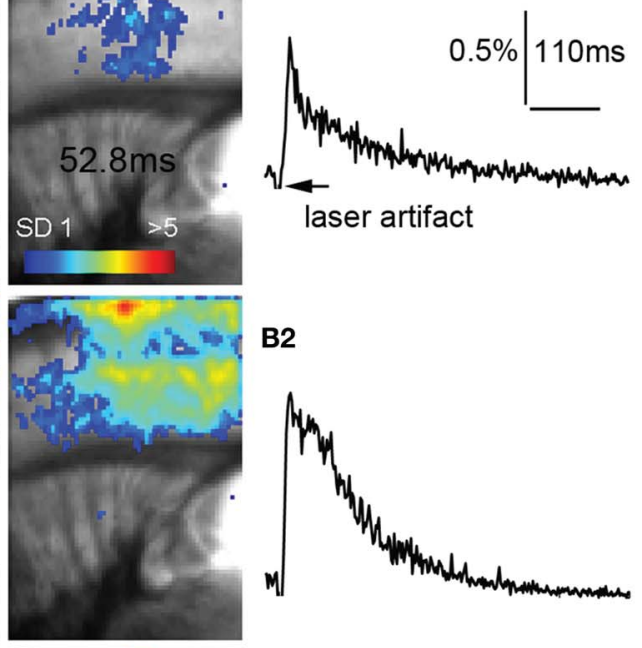

B2
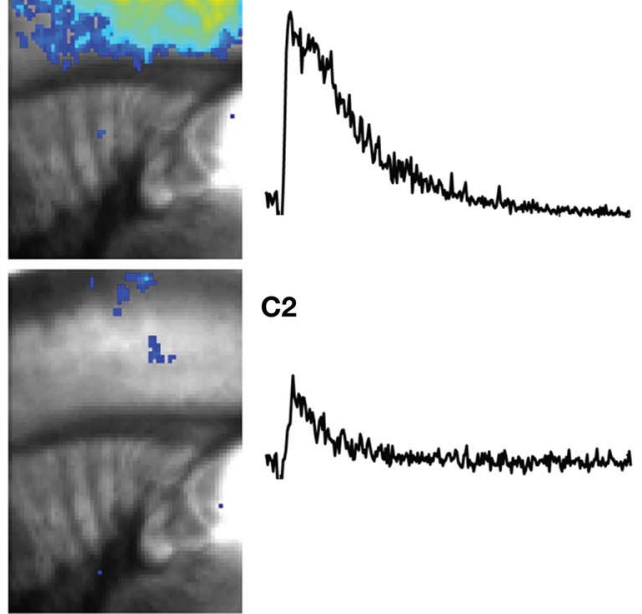

C2

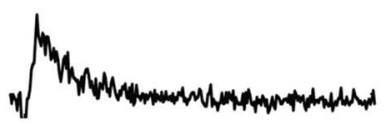

FIGURE 5 | AlstR-mediated inhibition of GABAergic neurons increases excitatory population activity in a DIx5/6-Cre: AlstR mouse slice.

(A1-C1) Time series data of VSD imaging of photostimulation-evoked activity in $\mathrm{S} 1$ cortex, in normal ACSF, in the presence and after washout of $A L$, respectively. The site of photostimulation is in cortical layer 4. (A2-C2) The time course of VSD signal (in the percent change of pixel intensity $[\Delta / / / \%]$ ) from the ROI indicated by the small rectangle in the first image frame in (A1-C1), respectively. 


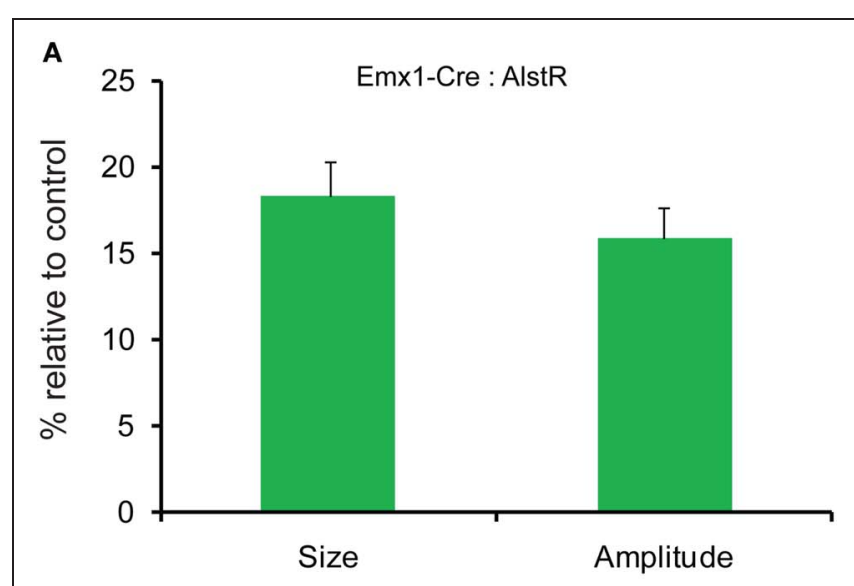

B

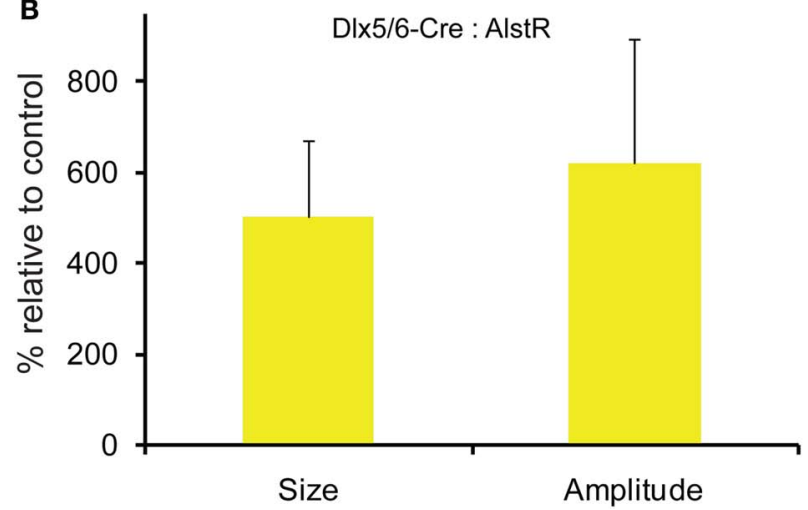

FIGURE 6 | Quantification of photostimulation-evoked responses in Emx1-Cre: AlstR and DIx5/6-Cre: AlstR mouse slices. (A) Bar graph indicating the average activation size and response amplitude in the presence of AL for Emx1-Cre: AlstR slices $(N=4)$, relative to normal ACSF control. (B) Bar graph indicating the average activation size and response amplitude in the presence of AL for DIx5/6-Cre: AlstR slices $(N=4)$, relative to normal ACSF control.

neurons. The experiments with single-cell electrophysiology, laser scanning photostimulation, and VSD imaging established that the AlstR-mediated inactivation of both excitatory and inhibitory neurons was specific and robust at single-cell and population levels. Our data also indicate that the AlstR system has good potential to be used as a molecular anticonvulsant for fast seizure control via cell-type specific expression.

As further demonstrated in this study, the AlstR/AL system has the required merits and applicable features as a potent molecular tool for regulation of cortical excitability. Optical inactivation of neurons can be effectively achieved by using the halorhodopsin or Arch system, which activates chloride or proton pumps and offers high temporal precision (Han and Boyden, 2007; Chow et al., 2010). Although no ligand is needed, prolonged optical silencing over the course of several minutes causes photo-toxicity and neuronal damage. In addition, optical illumination may interfere with functional imaging studies. One existing approach similar to the use of the non-native AlstR system is to genetically engineer G-protein-coupled receptors (GPCRs) of mammalian neurons (e.g., Receptors Activated Solely by Synthetic Ligands, or RASSLs) (Conklin et al., 2008; Alexander et al., 2009). For example, muscarinic acetylcholine receptors are engineered to generate a family of GPCRs that are activated solely by a pharmacologically inert drug-like compound (clozapine-N-oxide). The Gi-coupled designer receptor (hM4D) demonstrates its ability to induce membrane hyperpolarization and neuronal silencing in vitro and in vivo (Armbruster et al., 2007; Ferguson et al., 2011). The designed drug ligands can be administered in the periphery and cross the blood-brain barrier (BBB) to access receptors in deep brain structures and/or widely distributed neuronal populations. However, continued improvements are needed for the use of RASSLs, as the engineered receptors may be activated by non-selective, pharmacologically active small molecules. Furthermore, in some tissues RASSLs exhibit high levels of constitutive signaling in the absence of designed small-molecule agonists (Alexander et al., 2009), while the AlstR does not display evidence of basal signaling. Although the AL peptide is unlikely to cross the BBB in the current form and needs to be directly infused into brain tissue, future translational studies using the $\mathrm{AL}$ system will necessitate that AlstR ligands be designed with a capability of crossing the BBB. Systemic administration would likely result in some loss of temporal control, but it may still be sufficient to suppress excitatory activity of AlstR-expressing neurons. Please note that while the techniques for making BBB-permeable peptide analogs are established (Bickel et al., 2001; Egleton and Davis, 2005), the AL analogs for realistic human therapy will have to be developed toward this end.

A major focus of this work was to examine if the AlstR system can regulate the activity of diverse types of inhibitory cortical interneurons. Overall, inhibitory neurons can be inactivated, but compared to excitatory neurons, the degree of AlstR-mediated inhibition depended on interneuronal subtypes, which should be related to the factors including intrinsic neuronal excitability, AlstR expression levels, and endogenous GIRK densities. Given the availability of new interneuron-specific Cre driver lines developed by a NIH Neuroscience Blueprint Project in the laboratory of Josh Huang (available from the Jackson Laboratory) (Taniguchi et al., 2011), AlstR expression can be targeted to specific subsets of inhibitory cortical neurons for selectively suppressing their activity for future functional and behavioral studies.

In addition to the transgenic mouse approach, viral vectors can also be used for AlstR delivery in defined cell-types. Genetically modified adeno-associated viruses (AAV) and lentiviruses have been used for gene therapy in humans, enabling correcting certain genetic disorders in clinical trials (Davidson and Breakefield, 2003; Kootstra and Verma, 2003). The viral vectors can yield stable gene expression for months or years without apparent toxicity. Compared with the transgenic approach, major advantages of viral transfection include restricted expression in defined cortical regions, stronger AlstR expression levels with high-tier viruses, and the application to other species that transgenic approaches are impractical.

The AlstR approach represents an important advance for genetic manipulation of neuronal activity and can be valuable for many basic applications [see further discussion in (Callaway, 2005; Tan et al., 2006)]. We believe that the AlstR system can also 

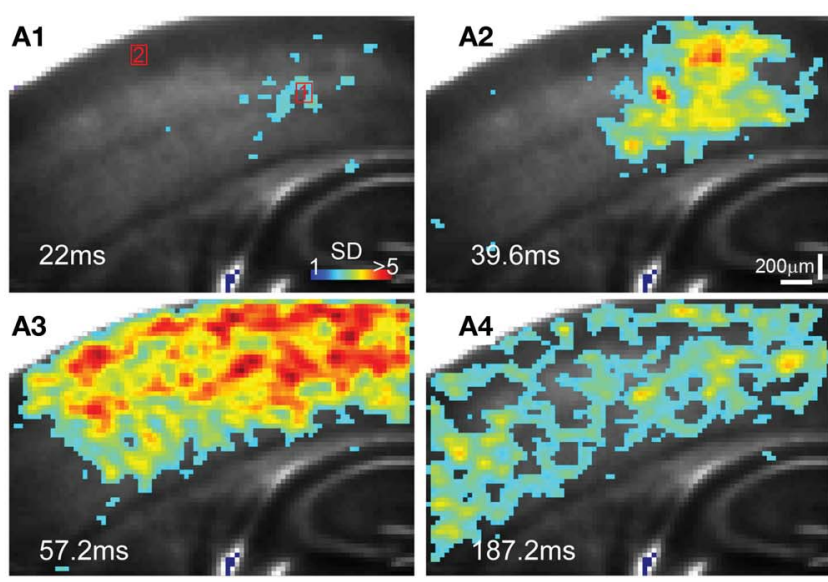

$39.6 \mathrm{~ms}$

17.

$200 \mu \mathrm{ml}$

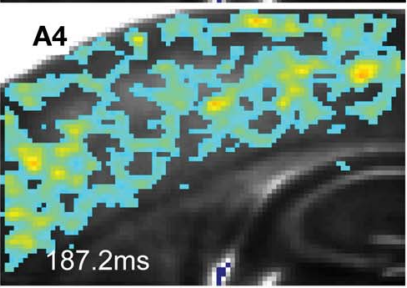

B1 ROI 1

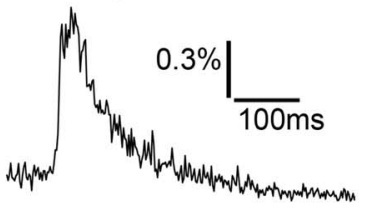

B2

$\mathrm{ROI} 2$

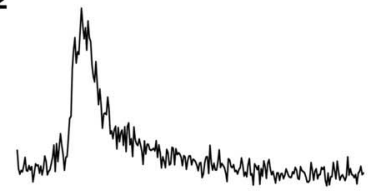

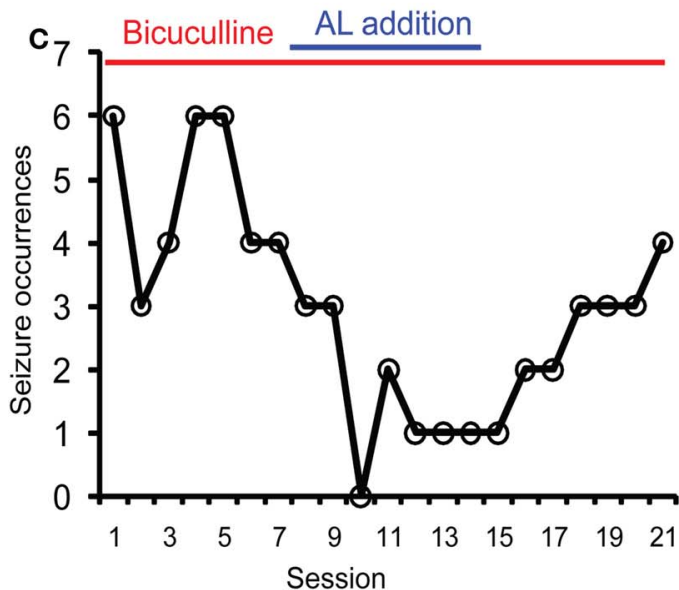

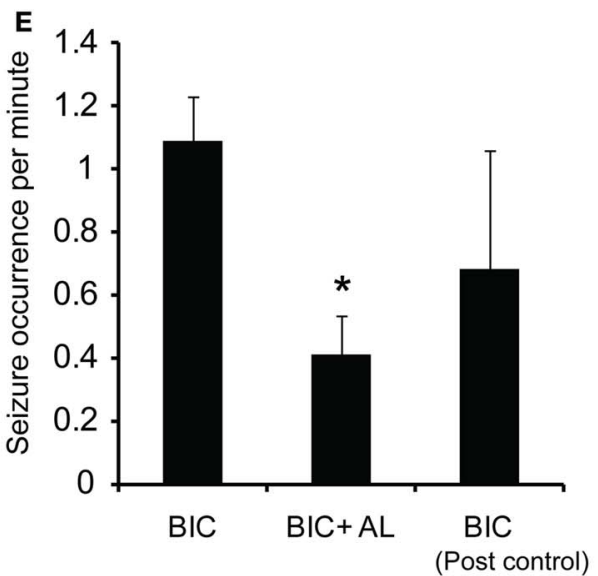

FIGURE 7 | The AlstR system suppresses the occurrence of bicuculline-induced spontaneous seizure-like activity. (A1-A4) Time series data of VSD imaging of bicuculline-induced spontaneous seizure-like activity in a Emx1-Cre: AlstR mouse slice. Specific time from the estimated seizure onset is labeled on the image. The color scale codes VSD signal amplitude expressed as SD multiples above the mean baseline. (B1, B2) The time course of VSD signal (in the percent change of pixel intensity $[\Delta / / / \%]$ ) from the two ROls (1 and 2) marked in $\mathbf{A} \mathbf{1}$ is plotted, respectively. Note that ROI 1 is close to the seizure initiation site. (C, D) The plots of seizure occurrences per imaging session (126 seconds per session)

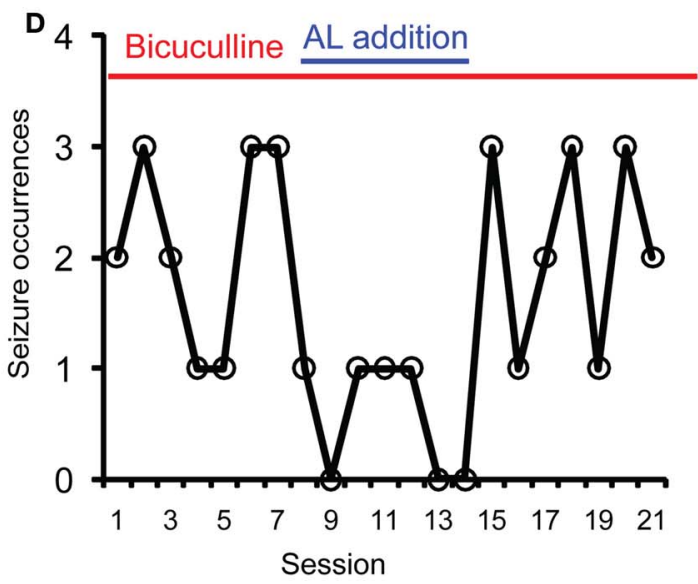

in the presence of bicuculline, bicuculline and $A L$, and bicuculline again for two different example slices. Each imaging session consisted of nine consecutive trials of sampling 8.8 seconds per trial (with a 14 seconds inter-trial interval); the number of seizure events was measured for each session. A interval of 15-20 minutes was allowed for the $A L$ infusion and washout before the next condition continued. (E) The data summary ( $N=5$ slices) showing that the co-application of $A L$ with bicuculline $(B I C)$ reduced the occurrence of bicuculline-induced seizure activity in Em×1-Cre: AlstR mouse slices. *Indicates statistical significance of $p<0.01$ between $\mathrm{BIC}$ and $\mathrm{BIC}+\mathrm{AL}$. 
be potentially used in translational applications, for example, in seizure control and epilepsy treatment. Despite the progress in antiepileptic drug development and surgical management, in about $30-40 \%$ of cases epileptic seizures do not respond well to the conventional therapies. We should capitalize on the development and use of new molecular and genetic tools to counteract seizure activity. Based upon the specificity and effectiveness of the

\section{REFERENCES}

Alexander, G. M., Rogan, S. C., Abbas, A. I., Armbruster, B. N., Pei, Y., Allen, J. A., Nonneman, R. J., Hartmann, J., Moy, S. S., Nicolelis, M. A., McNamara, J. O., and Roth, B. L. (2009). Remote control of neuronal activity in transgenic mice expressing evolved G protein-coupled receptors. Neuron 63, 27-39.

Armbruster, B. N., Li, X., Pausch, M. H., Herlitze, S., and Roth, B. L. (2007). Evolving the lock to fit the key to create a family of $G$ proteincoupled receptors potently activated by an inert ligand. Proc. Natl. Acad. Sci. U.S.A. 104, 5163-5168.

Bickel, U., Yoshikawa, T., and Pardridge, W. M. (2001). Delivery of peptides and proteins through the blood-brain barrier. Adv. Drug Deliv. Rev. 46, 247-279.

Birgul, N., Weise, C., Kreienkamp, H. J., and Richter, D. (1999). Reverse physiology in drosophila: identification of a novel allatostatin-like neuropeptide and its cognate receptor structurally related to the mammalian somatostatin/galanin/opioid receptor family. EMBO J. 18, 5892-5900.

Boyden, E. S., Zhang, F., Bamberg, E., Nagel, G., and Deisseroth, K. (2005). Millisecond-timescale, genetically targeted optical control of neural activity. Nat. Neurosci. 8, 1263-1268.

Callaway, E. M. (2005). A molecular and genetic arsenal for systems neuroscience. Trends Neurosci. 28, 196-201.

Chao, H. T., Chen, H., Samaco, R. C., Xue, M., Chahrour, M., Yoo, J., Neul, J. L., Gong, S., Lu, H. C., Heintz, N., Ekker, M., Rubenstein, J. L., Noebels, J. L., Rosenmund, C., and Zoghbi, H. Y. (2010). Dysfunction in GABA signalling mediates autism-like stereotypies and Rett syndrome phenotypes. Nature 468, 263-269.

Chow, B. Y., Han, X., Dobry, A. S., Qian, X., Chuong, A. S., Li, M., Henninger, M. A., Belfort, G. M., Lin, Y., Monahan, P. E., and Boyden, E. S. (2010). High-performance genetically targetable optical neural silencing by light-driven proton pumps. Nature $463,98-102$.
Conklin, B. R., Hsiao, E. C., Claeysen, S., Dumuis, A., Srinivasan, S., Forsayeth, J. R., Guettier, J. M., Chang, W. C., Pei, Y., McCarthy, K. D., Nissenson, R. A., Wess, J., Bockaert, J., and Roth, B. L. (2008). Engineering GPCR signaling pathways with RASSLs. Nat. Methods 5 , 673-678.

Davidson, B. L., and Breakefield, X. O. (2003). Viral vectors for gene delivery to the nervous system. Nat. Rev. Neurosci. 4, 353-364.

Egleton, R. D., and Davis, T. P. (2005). Development of neuropeptide drugs that cross the blood-brain barrier. NeuroRx 2, 44-53.

Ferguson, S. M., Eskenazi, D., Ishikawa, M., Wanat, M. J., Phillips, P. E., Dong, Y., Roth, B. L., and Neumaier, J. F. (2011). Transient neuronal inhibition reveals opposing roles of indirect and direct pathways in sensitization. Nat. Neurosci. 14, $22-24$.

Gorski, J. A., Talley, T., Qiu, M., Puelles, L., Rubenstein, J. L., and Jones, K. R. (2002). Cortical excitatory neurons and glia, but not GABAergic neurons, are produced in the Emxlexpressing lineage. J. Neurosci. 22, 6309-6314.

Gosgnach, S., Lanuza, G. M., Butt, S. J., Saueressig, H., Zhang, Y., Velasquez, T., Riethmacher, D., Callaway, E. M., Kiehn, O., and Goulding, M. (2006). V1 spinal neurons regulate the speed of vertebrate locomotor outputs. Nature 440, 215-219.

Guo, H., Hong, S., Jin, X. L., Chen, R. S., Avasthi, P. P., Tu, Y. T., Ivanco, T. L., and Li, Y. (2000). Specificity and efficiency of Cre-mediated recombination in Emx1-Cre knock-in mice. Biochem. Biophys. Res. Commun. 273, 661-665.

Han, X., and Boyden, E. S. (2007). Multiple-color optical activation, silencing, and desynchronization of neural activity, with single-spike temporal resolution. PLoS One 2, e299. doi: 10.1371/journal.pone.0000299

Karpova, A. Y., Tervo, D. G., Gray, N. W., and Svoboda, K. (2005). Rapid and reversible chemical inactivation of synaptic transmission in genetically targeted neurons. Neuron 48, 727-735.

AlstR system, this genetic inactivation system can be potentially developed as a molecular anticonvulsant with few side effects.

\section{ACKNOWLEDGMENTS}

This work was funded by the US National Institutes of Health grants DA023700 and DA023700S1 to Xiangmin Xu. We thank Nicholas Olivas for helpful comments on the manuscript.

Karschin, C., Dissmann, E., Stuhmer, W., and Karschin, A. (1996). IRK (1-3) and GIRK(1-4) inwardly rectifying $\mathrm{K}+$ channel mRNAs are differentially expressed in the adult rat brain. J. Neurosci. 16, 3559-3570.

Kim, J. C., Cook, M. N., Carey, M. R., Shen, C., Regehr, W. G., and Dymecki, S. M. (2009). Linking genetically defined neurons to behavior through a broadly applicable silencing allele. Neuron 63, 305-315.

Kootstra, N. A., and Verma, I. M. (2003). Gene therapy with viral vectors. Annu. Rev. Pharmacol. Toxicol. 43, 413-439.

Kuhlman, S. J., and Huang, Z. J. (2008). High-resolution labeling and functional manipulation of specific neuron types in mouse brain by Cre-activated viral gene expression. PLoS One 3, e2005. doi: 10.1371/journal.pone.0002005

Laaris, N., Carlson, G. C., and Keller, A. (2000). Thalamic-evoked synaptic interactions in barrel cortex revealed by optical imaging. $J$. Neurosci. 20, 1529-1537.

Lechner, H. A., Lein, E. S., and Callaway, E. M. (2002). A genetic method for selective and quickly reversible silencing of mammalian neurons. J. Neurosci. 22, 5287-5290.

Lerchner, W., Xiao, C., Nashmi, R. Slimko, E. M., van Trigt, L., Lester, H. A., and Anderson, D. J. (2007) Reversible silencing of neuronal excitability in behaving mice by a genetically targeted, ivermectingated Cl- channel. Neuron 54, 35-49.

Luo, L., Callaway, E. M., and Svoboda, K. (2008). Genetic dissection of neural circuits. Neuron 57, 634-660.

Madisen, L., Zwingman, T. A., Sunkin, S. M., Oh, S. W., Zariwala, H. A., Gu, H., Ng, L. L., Palmiter, R. D., Hawrylycz, M. J., Jones, A. R., Lein, E. S., and Zeng, H. (2010). A robust and high-throughput Cre reporting and characterization system for the whole mouse brain. Nat. Neurosci. 13, 133-140.

Monory, K., Massa, F., Egertová, M., Eder, M., Blaudzun, H., Westenbroek, R., Kelsch, W., Jacob, W., Marsch, R., Ekker, M., Long, J., Rubenstein, J. L., Goebbels, S., Nave, K. A., During, M.,
Klugmann, M., Wölfel, B., Dodt, H. U., Zieglgänsberger, W., Wotjak, C. T., Mackie, K., Elphick, M. R., Marsicano, G., and Lutz, B. (2006). The endocannabinoid system controls key epileptogenic circuits in the hippocampus. Neuron 51, 455-466.

Panganiban, G., and Rubenstein, J. L. (2002). Developmental functions of the Distal-less/Dlx homeobox genes. Development 129, 4371-4386.

Petersen, C. C., and Sakmann, B. (2001). Functionally independent columns of rat somatosensory barrel cortex revealed with voltagesensitive dye imaging. J. Neurosci. 21, 8435-8446.

Rodriguez, C. I., Buchholz, F., Galloway, J., Sequerra, R., Kasper, J., Ayala, R., Stewart, A. F., and Dymecki, S. M. (2000). Highefficiency deleter mice show that FLPe is an alternative to Cre-loxP. Nat. Genet. 25, 139-140.

Tan, E. M., Yamaguchi, Y., Horwitz, G. D., Gosgnach, S., Lein, E. S., Goulding, M., Albright, T. D., and Callaway, E. M. (2006). Selective and quickly reversible inactivation of mammalian neurons in vivo using the Drosophila allatostatin receptor. Neuron 51, 157-170.

Tan, W., Janczewski, W. A., Yang, P., Shao, X. M., Callaway, E. M., and Feldman, J. L. (2008). Silencing preBotzinger complex somatostatinexpressing neurons induces persistent apnea in awake rat. Nat. Neurosci. 11, 538-540.

Taniguchi, H., He, M., Wu, P., Kim, S., Paik, R., Sugino, K., Kvitsani, D., Fu, Y., Lu, J., Lin, Y., Miyoshi, G., Shima, Y., Fishell, G., Nelson, S. B., and Huang, Z. J. (2011). A resource of Cre driver lines for genetic targeting of GABAergic neurons in cerebral cortex. Neuron 71, 995-1013.

Wehr, M., Hostick, U., Kyweriga, M., Tan, A., Weible, A. P., Wu, H., Wu, W., Callaway, E. M., and Kentros, C. (2009). Transgenic silencing of neurons in the mammalian brain by expression of the allatostatin receptor (AlstR). J. Neurophysiol. 102, 2554-2562.

Xu, X. (2011). "High precision and fast functional mapping of brain circuitry through laser scanning photostimulation and fast dye 
imaging," in Laser Scanning, Theory and Applications, ed C.-C, Wang (Rijeka, Croatia: InTech), 113-132.

Xu, X., Olivas, N. D., Levi, R., Ikrar, T., and Nenadic, Z. (2010). High precision and fast functional mapping of cortical circuitry through a combination of voltage sensitive dye imaging and laser scanning photostimulation. J. Neurophysiol. 103, 2301-2312.

Conflict of Interest Statement: The authors declare that the research was conducted in the absence of any commercial or financial relationships that could be construed as a potential conflict of interest.
Received: 29 September 2011; accepted: 02 January 2012; published online: 20 January 2012.

Citation: Ikrar T, Shi Y, Velasquez T, Goulding M and Xu X (2012) Cell-type specific regulation of cortical excitability through the allatostatin receptor system. Front. Neural Circuits 6:2. doi: 10.3389/ fncir.2012.00002
Copyright (C) 2012 Ikrar, Shi, Velasquez, Goulding and $\mathrm{Xu}$. This is an openaccess article distributed under the terms of the Creative Commons Attribution Non Commercial License, which permits non-commercial use, distribution, and reproduction in other forums, provided the original authors and source are credited. 